\title{
The Two-Component Majorana Equation-Novel Derivations and Known Symmetries
}

\author{
Eckart Marsch \\ Max-Planck-Institut für Sonnensystemforschung, Katlenburg-Lindau, Germany \\ E-mail:marsch@mps.mpg.de \\ Received May 26, 2011; revised July 10, 2011; accepted July 25, 2011
}

\begin{abstract}
We revisit the two-component Majorana equation and derive it in a new form by linearizing the relativistic dispersion relation of a massive particle, in a way similar to that used to derive the Dirac equation. We are using thereby the Pauli spin matrices, corresponding to an irreducible representation of the Lorentz group, and a lucid and transparent algebraic approach exploiting the newly introduced spin-flip operator. Thus we can readily build up the Majorana version of the Dirac equation in its chiral representation. The Lorentz-invariant complex conjugation operation involves the spin-flip operator, and its connection to chiral symmetry is discussed. The eigenfunctions of the Majorana equation are calculated in a concise way.
\end{abstract}

Keywords: Dirac Equation, Majorana Equation, Charge Conjugation, Chiral Symmetry

\section{Introduction}

The so called two-component Majorana equation [1,2] permitting a finite mass term has been used in modern quantum field theory for the description of massive neutrinos, ever since convincing empirical evidence [3] for their finite masses and associated oscillations has been found in the past decade. Yet, in the standard model of elementary particle physics (see, e.g., text books $[3,4]$ ) the fermions involved still are assumed to be massless, and thus obey chiral symmetry. The corresponding neutrinos are conventionally described by the massless Weyl [5] equation involving two-component Pauli [6] spinors. As is well known, the Weyl equation corresponds to two possible irreducible representations of the Lorentz group.

In this paper we present among other topics a novel derivation of the two-component Majorana equation with a mass term, whereby we linearize the relativistic dispersion relation of a massive particle in a way similar to that used in deriving the Dirac equation. Moreover, a consistent interpretation for the Dirac equation is given concerning its property of covariant complex conjugation [7]. It emerges as an intrinsic basic symmetry of the reducible Dirac equation with its four-component spinors, and includes of course the charge exchange operation for the Dirac equation [8] when being coupled to a gauge field like the electromagnetic field. We use a concise algebraic nomenclature for the Dirac equation, and in a lucid way present its classical symmetries such as parity, time reversal and charge exchange.

\section{Dirac Equation in a Concise Algebraic Form}

In this somewhat tutorial subsection, we consider the Dirac equation in the standard and chiral representations. The derivation of the Dirac equation can be found in any textbook on quantum field theory (e.g., the ones of Kaku [5] or Das [9]). We use standard symbols, notations and definitions, and conventionally use units of $\hbar=c=1$. The Dirac Equation [8] generally reads

$$
\gamma^{\mu} p_{\mu} \psi=\mathrm{i} \gamma^{\mu} \partial_{\mu} \psi=m \psi
$$

with $p_{\mu}=\left(p_{0},-\boldsymbol{p}\right)=i \partial_{\mu}=i(\partial / \partial t, \partial / \partial \boldsymbol{x})$ being the fourmomentum operator acting on the spinor wave function $\psi(\boldsymbol{x}, t)$. The particle mass is $m$, and the four-vector named as usually $\gamma^{\mu}$ is composed of the four Dirac matrices. They can concisely be expressed in terms of the spin operator $\sigma$ and the three operators $\alpha, \beta$ and $\gamma$ to be defined in their matrix form below. The three Pauli matrices, to which we may add the $2 \times 2$ unit matrix denoted as $\sigma_{0}$, have standard form,

$$
\sigma_{\mathrm{x}}=\left(\begin{array}{ll}
0 & 1 \\
1 & 0
\end{array}\right), \sigma_{\mathrm{y}}=\left(\begin{array}{cc}
0 & -i \\
i & 0
\end{array}\right), \sigma_{\mathrm{z}}=\left(\begin{array}{cc}
1 & 0 \\
0 & -1
\end{array}\right)
$$


and build the three-vector $\sigma=\left(\sigma_{\mathrm{x}}, \sigma_{\mathrm{y}}, \sigma_{\mathrm{z}}\right)$, by help which we can introduce the two four-vector forms of the Pauli matrices, which we may refer to as Weyl matrices. They are defined by the four vectors

$$
\sigma_{ \pm}^{\mu}=\left(\sigma_{0}, \pm \sigma\right)
$$

and obey $\sigma_{ \pm}^{\mu}=\sigma_{\mp \mu}$. Similarly to the Pauli matrices, we introduce the three real matrices

$$
\alpha=\left(\begin{array}{ll}
0 & 1 \\
1 & 0
\end{array}\right), \beta=\left(\begin{array}{cc}
1 & 0 \\
0 & -1
\end{array}\right), \gamma=\left(\begin{array}{cc}
0 & 1 \\
-1 & 0
\end{array}\right)
$$

Note that $\gamma=\beta \alpha$, and that all three matrices mutually anti-commute with each other, just like the Pauli matrices do. All what we need in the following are the algebraic properties of the operators involved, like $\alpha \beta+\beta \alpha=0, \alpha \gamma+\gamma \alpha=0$, and $\beta \gamma+\gamma \beta=0$, and that $\alpha^{2}=\beta^{2}=1$ and $\gamma^{2}=-1$. Similarly, the spin operator components obey $\sigma_{\mathrm{x}} \sigma_{\mathrm{y}}+\sigma_{\mathrm{y}} \sigma_{\mathrm{x}}=0, \sigma_{\mathrm{x}} \sigma_{\mathrm{z}}+\sigma_{\mathrm{z}} \sigma_{\mathrm{x}}=0$, and $\sigma_{\mathrm{y}} \sigma_{\mathrm{z}}+\sigma_{\mathrm{z}} \sigma_{\mathrm{y}}=0$, and it is $\sigma_{\mathrm{x}}^{2}=\sigma_{\mathrm{y}}^{2}=\sigma_{\mathrm{z}}^{2}=1$. Furthermore, for any three vector $\boldsymbol{v}$ the relation $(\sigma \cdot v)^{2}=v^{2}$ holds. In terms of all these operators we can express the gamma four-vector as a direct product and thus rewrite it in the standard Dirac representation as

$$
\gamma^{\mu}=\left(\beta \sigma_{0}, \gamma \boldsymbol{\sigma}\right)
$$

Finally, we can concisely quote the Dirac equation with mass term in conventional Hamiltonian form as

$$
i\left(\frac{\partial}{\partial t}+\alpha \boldsymbol{\sigma} \cdot \frac{\partial}{\partial \boldsymbol{x}}\right) \psi(\boldsymbol{x}, t)=m \beta \psi(\boldsymbol{x}, t)
$$

Subsequently, we shall also work with the Dirac equation in its chiral representation, and therefore quote the relevant matrices here, which are given instead of (4) in the form

$$
\alpha=\left(\begin{array}{cc}
1 & 0 \\
0 & -1
\end{array}\right), \beta=\left(\begin{array}{ll}
0 & 1 \\
1 & 0
\end{array}\right), \gamma=\left(\begin{array}{cc}
0 & -1 \\
1 & 0
\end{array}\right)
$$

Here we used to ease the notation the same symbols as in the standard representation. There is another important matrix named $\gamma^{5}$, which (see for example the textbook of Kaku [4]) is defined as $\gamma^{5}=i \gamma^{0} \gamma^{1} \gamma^{2} \gamma^{3}$. Inserting the above matrices and the Pauli matrices we obtain,

$$
\gamma^{5}=i \beta \sigma_{0} \beta \alpha \sigma_{x} \beta \alpha \sigma_{y} \beta \alpha \sigma_{z}=\alpha \sigma_{0}
$$

Here we used the properties that $\sigma_{x} \sigma_{y} \sigma_{z}=i \sigma_{0}$, $\sigma_{0}^{2}=\sigma_{0}$, and $\alpha \beta=-\beta \alpha$. By means of $\gamma^{5}$ the chiral projection operators, $P_{ \pm}=1 / 2(1 \pm \alpha)$, can be written

$$
P_{+}=\left(\begin{array}{ll}
1 & 0 \\
0 & 0
\end{array}\right), \quad P_{-}=\left(\begin{array}{ll}
0 & 0 \\
0 & 1
\end{array}\right)
$$

We are subsequently going to consider the symmetry properties of the Dirac Equation (1), including chirality.

\section{New Derivation of the Majorana Equation}

In this section a novel derivation of the two-component Majorana equation [8] term is presented, by linearizing the standard dispersion relation of a massive relativistic particle. For this purpose, we have at the outset to define an important operator, which is not of pure algebraic nature but involves the complex-conjugation operator named as $C$. This antisymmetric operator called $S$ is defined as $S=i \sigma_{y} C$, and obeys the relations $S^{-1}=S^{\dagger}=-S$ and $S^{2}=-1$. The operation of $S$ on the spin vector $\boldsymbol{\sigma}$ leads to its inversion, i.e., the operation $S \sigma S^{-1}=-\sigma$ yields a spin flip. Also remember from Equation (2) that $\boldsymbol{\sigma}^{*}=\boldsymbol{\sigma}^{\mathrm{T}}$, where the superscript $\mathrm{T}$ indicates the transposed matrix. As $S$ flips the spin, we have $\sigma_{ \pm} S=S \sigma_{\mp}$, and $S i=-i S$ because of the action of $C$. Therefore, $S$ also anti-commutes with the momentum four vector $p_{\mu}$, and thus we have $S p_{0}+p_{0} S=0$ and $\boldsymbol{p} S+S \boldsymbol{p}=\mathbf{0}$.

Making use of the spin-flip operator $S$ one can derive the Majorana equation with a mass term in new and straightforward way from the relativistic energy and momentum relation which in fact is constitutive for all field equations, $E^{2}=m^{2}+\boldsymbol{P}^{2}$, where the four-momentum of the particle is $P^{\mu}=(E, \boldsymbol{P})$. The energy-momentum relation is usually written as mass-shell condition,

$$
P^{\mu} P_{\mu}=m^{2}
$$

Following Dirac [8], his Equation (1) results from a linearization of (10), which requires to go beyond the algebra of complex numbers and needs the introduction of his famous matrices $\alpha=\alpha \boldsymbol{\sigma}$ and $\beta$, yielding the Hamiltonian $\mathrm{H}=m \beta+\boldsymbol{\alpha} \cdot \boldsymbol{p}$. As shown in text books [4,5], one requires four-dimensional matrices to obtain the algebra of the Dirac matrices defined in Equations (2-5). The Pauli matrices (2) alone only suffice if there is no mass term, in which case one obtains the Weyl equation [5].

However, recalling the above properties of the spin flip operator, $S=i \sigma_{y} C$, we can overcome this problem and still use the two-component representation given by the Pauli matrices (2). Namely, by help of $S$ we can go a mathematical step beyond pure matrix algebra and define the linear but non-hermitian operator

$$
\mathrm{H}=m S+\boldsymbol{\sigma} \cdot \boldsymbol{p}
$$

When squaring this equation and multiplying it out, the key properties $S^{2}=-1, S \sigma+\sigma S=0$, and $S i+i S=0$ must be exploited. Because of this last relation, $p_{0}$ and $\boldsymbol{p}$ also anti-commute with $S$. Thus with $p_{0}=\mathrm{H}$ we obtain

$$
m^{2}=S\left(p_{0}-\boldsymbol{\sigma} \cdot \boldsymbol{p}\right) S\left(p_{0}-\boldsymbol{\sigma} \cdot \boldsymbol{p}\right)=p_{0}^{2}-(\boldsymbol{\sigma} \cdot \boldsymbol{p})^{2}
$$


which when inserting the differential operators explicitly yields the Klein-Gordon equation. Similarly, we obtain from (11) with $p_{0}=\mathrm{H}$ a linear wave equation (named after Weyl without the mass term), which involves only the Pauli matrix operators acting on a two-component spinor named $\phi$ and reads as follows:

$$
i\left(\frac{\partial}{\partial t}+\boldsymbol{\sigma} \cdot \frac{\partial}{\partial \boldsymbol{x}}\right) \phi(\boldsymbol{x}, t)=m S \phi(\boldsymbol{x}, t)
$$

This equation is nothing but the two-component Majorana equation [2], as it can be derived from Dirac's equation in chiral form, if one imposes on it the condition of Lorentz-covariant complex conjugation, a procedure which is clearly described in a recent tutorial paper of Pal [7].

Obviously, the Majorana Equation (13) is very sensitive to the operation $S$. Namely, when we apply it from the left side we find that

$$
i\left(\frac{\partial}{\partial t}-\boldsymbol{\sigma} \cdot \frac{\partial}{\partial \boldsymbol{x}}\right) S \phi(\boldsymbol{x}, t)=-m S(S \phi(\boldsymbol{x}, t))=m \phi(\boldsymbol{x}, t)
$$

Consequently, if $\phi$ solves the Equation (13) with the plus sign in front of $\boldsymbol{\sigma}$, then $S \phi$ solves it with the minus sign, but with a minus sign also at the mass term. Therefore, Equations (13) and (14) are closely connected, and represent only one independent complex spinor field, which can either be $\phi$ or $S \phi$. Instead of its two complex component fields we may consider four real fields, which correspond to the real and imaginary parts of the two components of $\phi$, and hence the name Majorana equation for (13), because it is equivalent to the real four-component representation of the Dirac spinor as obtained from (1) by a unitary transformation, a result which is explicitly shown in [3], for example.

Making again use of the spin-flip operator $S$, one can derive the Majorana equation with a mass term in yet another way from the relativistic energy-momentum mass-shell condition (10). The algebra of the newly defined four-vector $S^{\mu}=S\left(\sigma_{0}, \sigma\right)$ permits a representation that is equivalent to the Clifford algebra of the $\gamma^{\mu}$ matrices. Namely, we find a decomposition of the metric tensor $g^{\mu v}$ in the form,

$$
\left\{S^{\mu}, S^{\nu}\right\}=S^{\mu} S^{v}+S^{v} S^{\mu}=-2 g^{\mu v}
$$

which is validated by using the anti-commutator $\{S, \sigma\}=0$, and that $S^{2}=-1$. Note also that $S^{\mu} S_{\mu}=-4$. Consequently, we can write the mass shell condition (10) also in the form:

$$
P^{\mu} P_{\mu}=g^{\mu v} P_{\mu} P_{v}=-S^{\mu} S^{v} P_{\mu} P_{v}=m^{2}
$$

Here the momentum four-vector components were treated as real numbers.

Returning finally to Equation (13) again, we now derive its eigenfunctions. In order to solve (13), we make the usual plane-wave ansatz,

$\phi(\boldsymbol{x}, t)=u \exp (-i E t+i \boldsymbol{P} \cdot \boldsymbol{x})+v \exp (+i E t-i \boldsymbol{P} \cdot \boldsymbol{x})$. The resulting linked eigenspinor equations are

$$
\begin{gathered}
(E-\boldsymbol{\sigma} \cdot \boldsymbol{P}) u(\boldsymbol{P}, E)=m S v(\boldsymbol{P}, E) \\
(E-\boldsymbol{\sigma} \cdot \boldsymbol{P}) v(\boldsymbol{P}, E)=-m S u(\boldsymbol{P}, E)
\end{gathered}
$$

By insertion of the first into the second equation, or vice versa, the relativistic dispersion relation yields the two eigenvalues

$$
E_{1,2}(\boldsymbol{P})= \pm \sqrt{m^{2}+\boldsymbol{P}^{2}}
$$

which are obtained from the requirement for nontrivial solutions of the spinors $u$ and $v$ to exist. The negative root in (19) cannot be neglected, since as usually it is related to antiparticles. We can solve (17) for $v$ and insert it back into the above ansatz for $\phi(x, t)$ to obtain finally the solutions of (13) and (14) in the concise forms

$$
\begin{aligned}
& \phi(\boldsymbol{x}, t)=\left(1-\frac{E+\boldsymbol{\sigma} \cdot \boldsymbol{P}}{m} S\right) \exp (-i E t+i \boldsymbol{P} \cdot \boldsymbol{x}) u \\
& S \phi(\boldsymbol{x}, t)=\left(S+\frac{E-\boldsymbol{\sigma} \cdot \boldsymbol{P}}{m}\right) \exp (-i E t+i \boldsymbol{P} \cdot \boldsymbol{x}) u
\end{aligned}
$$

To validate these solutions by direct differentiation, careful attention must be paid to the anticommutation rules between $S$ and $i$, respectively $\boldsymbol{\sigma}$. We are free to choose for the eigenspinor $u$ the two standard spin up and down eigenfunctions: $u_{1}^{\dagger}=(1,0)$ and $u_{2}^{\dagger}=(0,1)$. Similar solutions like that of Equations (20) and (21) are obtained for the negative energy root in (19), yielding the antiparticle wavefunctions. Superposition of all these Fourier modes and their summation over the momentum variable $\boldsymbol{P}$ leads finally to the general solution of the Majorana fields, whose quantization then readily follows from the canonical rules [2-4,9].

\section{Symmetries of the Dirac Equation Revisited}

Let us now consider the symmetries of the Dirac equation, in particular the charge exchange $C$, parity $P$, and time reversal $T$ operations. Generally speaking the Dirac equation is invariant under the symmetry operation $\mathrm{O}$, if the spinor

$$
\psi^{\mathrm{O}}=\mathrm{O} \psi
$$

also fulfils the Dirac equation. Therefore, when applying the operation $\mathrm{O}$ from the left and its inverse $\mathrm{O}^{-1}$ from the right, whereby the unit operator is given by the 
decomposition $\mathrm{OO}^{-1}=1$, we obtain the result

$$
\left(\mathrm{O}\left(i \frac{\partial}{\partial t}+i \alpha \boldsymbol{\sigma} \cdot \frac{\partial}{\partial \boldsymbol{x}}\right) \mathrm{O}^{-1}-m \mathrm{O} \beta \mathrm{O}^{-1}\right) \mathrm{O} \psi(\boldsymbol{x}, t)=0
$$

We still need to define the time and space coordinate inversion operations $T$ and $P$ on a spinor $\psi$ by

$$
\begin{aligned}
& T \psi(\boldsymbol{x}, t)=\psi(\boldsymbol{x},-t) \\
& P \psi(\boldsymbol{x}, t)=\psi(-\boldsymbol{x}, t)
\end{aligned}
$$

and also recall the complex conjugation operation $C$, which gives $\mathrm{CiC}^{-1}=-i$, and yields

$$
C \psi(\boldsymbol{x}, t)=\psi^{*}(\boldsymbol{x}, t)
$$

Here the asterisk denotes as usually the complex conjugate number. In what follows we do not always quote explicitly the unit matrix $\sigma_{0}$, or the unit matrix simply named 1 , which is associated with the matrices in (4) and (7). With all these preparations in mind, it is easy to see which operators provide the requested symmetry operations. We compose them in the Table 1. To complete the operator algebra, it is important to note the following commutation relations. Of course, the coordinate reversal operators $T$ and $P$ commute with $\alpha, \beta$ and $\gamma$, and alike with the three Pauli matrices. These in turn anti-commute with $S$, but obey $[\alpha, \boldsymbol{\sigma}]=[\beta, \boldsymbol{\sigma}]=[\gamma, \boldsymbol{\sigma}]=\mathbf{0}$, with the commutator denoted by $[$,$] .$

Let us first consider in (23) the time reversal, $\mathrm{O}=\mathrm{T}=S T$. Apparently, it does not affect the mass term with $\beta$, and also leaves the first term as well as i $\boldsymbol{\sigma}$ and $\alpha$ unchanged. Therefore, also $\psi^{\top}=i \sigma_{y} \psi^{*}(\boldsymbol{x},-t)$ solves the Dirac Equation (23). Similarly, the parity operation $\mathrm{O}=\mathrm{P}=\beta P$ commutes with the mass term and does not affect the first term in (23). But it also leaves the momentum term invariant since $\alpha$ and $\boldsymbol{x}$ both change signs together. Therefore, also

$\psi^{P}=\beta \psi(-\boldsymbol{x}, t)$ solves the Dirac equation. Finally, we introduce charge conjugation as $\mathrm{O}=\mathrm{C}=\gamma S$, which changes the signs of all three terms in (23) with no net effect, yet thereby leaves the kinetic term $\alpha \boldsymbol{\sigma}$ invariant as well. Therefore, also $\psi^{\mathrm{C}}=\gamma i \sigma_{y} \psi^{*}(\boldsymbol{x}, t)$ solves the Dirac equation. In conclusion, the symmetry operations of Table 1 work in a transparent and simple way on the Dirac equation. We finally quote the charge conjugation operator explicitly in chiral matrix form (7) as

Table 1. Symmetry operations.

\begin{tabular}{cccc}
\hline Operation & Time reversal & Parity & Charge conjugation \\
\hline Operator & $\mathrm{T}=S T$ & $\mathrm{P}=\beta P$ & $\mathrm{C}=\gamma S$ \\
\hline
\end{tabular}

$$
\mathrm{C}=\left(\begin{array}{cc}
0 & -S \\
S & 0
\end{array}\right)
$$

In the standard representation (4) we simply get the transposed matrix. Consequently, $\mathrm{C}^{2}=-S^{2}=1$, as it should be. Traditionally, the operator $\mathrm{C}$ is associated with charge conjugation, because the effect it has on the Dirac equation when coupled to a gauge field, like the electromagnetic four-vector potential $A_{\mu}$, is simply that the sign of the charge is changed. As the minimal coupling $[4,9]$ to the field (the particle charge is $e$ ) yields the replacement,

$$
\partial_{\mu} \Rightarrow \partial_{\mu}+i e A_{\mu}
$$

the effect of $\mathrm{C}$ will, solely due to the action of $C$, be the substitution of $e$ by $-e$. However, what then is the meaning of $\mathrm{C}$ for the free Dirac equation, when not being coupled to a gauge field?

\section{Chiral Symmetry and Complex Conjugation}

Again, what is the genuine symmetry the operation $\mathrm{C}$ is related with? The answer has been given in a most lucid way by Pal [7] in his recent paper. The operation C in fact is the Lorentz-covariant complex conjugation, which is an operation that of course makes sense without even considering gauge fields. However, the operator $\boldsymbol{\sigma}$ plays a key role not only in this issue, but also for the two irreducible representations of the Lorentz group related with two-component Pauli spinors. We recall the operator $S$ causes the spin $\boldsymbol{\sigma}$ to flip and inverts its direction. The parity operation does not change the sign of $\boldsymbol{\sigma}$ being a pseudo vector, which is consistent with the required invariance of the spin angular momentum commutator, $\sigma \times \sigma=2 i \sigma$, under parity. So the intrinsic symmetry described by $\mathrm{C}$ is closely connected with the degeneracy of the Dirac equation (with its fourcomponent spinors) with respect to a spin flip, which corresponds to interchanging the two irreducible representations of the Lorentz group. As a consequence, we can combine the two interrelated Majorana Equations (13) and (14), and thus construct from them the fourcomponent spinor

$$
\psi^{\mathrm{C}}=\left(\begin{array}{c}
\phi \\
S \phi
\end{array}\right)
$$

How does this state $\psi^{\mathrm{C}}$ transform under the operation $C$ ? We apply the charge-conjugation or covariant complex conjugation operation in its matrix form in (27) on this special spinor and then obtain 


$$
\mathrm{C} \psi^{\mathrm{C}}=\left(\begin{array}{cc}
0 & -S \\
S & 0
\end{array}\right) \cdot\left(\begin{array}{c}
\phi \\
S \phi
\end{array}\right)=\left(\begin{array}{c}
\phi \\
S \phi
\end{array}\right)=\psi^{\mathrm{C}}
$$

Consequently, the charge-conjugation operator leaves the special state $\psi^{\mathrm{C}}$ unchanged, which therefore is an eigenfunction with eigenvalue unity.

We recall here Dirac's Equation (6) in its chiral matrix form:

$$
\gamma^{\mu} p_{\mu} \psi=\left(\begin{array}{cc}
0 & \sigma_{-}^{\mu} \\
\sigma_{+}^{\mu} & 0
\end{array}\right) i \partial_{\mu} \psi=m \psi
$$

Apparently, any chiral Dirac spinor can be decomposed into two-component Pauli spinors such that $\psi^{\dagger}=\left(\phi_{+}^{\dagger}, \phi_{-}^{\dagger}\right)$, where the plus sign indicates the right-chiral and minus sign the left-chiral components of the original Dirac spinor. We recall the chiral projection operators defined in (9), which yield the spinors $\psi_{\mathrm{R}}=P_{+} \psi=\left(\phi_{+}, 0\right)^{\dagger}$ and $\psi_{\mathrm{L}}=P_{-} \psi=\left(0, \phi_{-}\right)^{\dagger}$.

Now by comparison with equation (29) we have the obvious connections, $\phi_{+}=\phi$ and $\phi_{-}=S \phi$. Therefore, a comparison with the Majorana Equations (13) and (14) shows that $\psi^{\mathrm{C}}$ fulfils the chiral Dirac equation, but it is special in so far as it also is an eigenfunction of the charge-conjugation operator, which is to say the covariant complex conjugation operator $\mathrm{C}$. Because of this constraint the spinor (29) is determined fully by the two-component spinor $\phi$ that obeys the Majorana Equation (13). If we then apply $C$ separately to the right- or left-chiral fields we obtain

$$
\mathrm{C}\left[\left(\psi^{\mathrm{C}}\right)_{\mathrm{R}}\right]=\left(\psi^{\mathrm{C}}\right)_{\mathrm{L}} \text { and } \mathrm{C}\left[\left(\psi^{\mathrm{C}}\right)_{\mathrm{L}}\right]=\left(\psi^{\mathrm{C}}\right)_{\mathrm{R}}
$$

Thus this operator exchanges the chirality of the chiral projections of the complex-self-conjugate spinor $\psi^{\mathrm{C}}$, and it transforms left- into right-handed states and vice versa, a virtue which is essentially associated with the spin flip operator $S$ defining C. In conclusion, in this special situation the operator $\mathrm{C}$ acts as chirality conjugation or reversal. While being composed of the two irreducible Majorana equations, the reducible chiral Dirac equation has as an intrinsic symmetry this chirality conjugation. The same chirality reversal is obtained for the real four-component Majorana equation, in which complex conjugation of the chiral eigenspinors changes the left- into right-chiral spinors and vice versa.

When we use the standard Dirac representation with the matrices of (4), the standard Dirac spinor can also be decomposed into two-component Pauli spinors such that $\psi^{\dagger}=\left(\phi_{1}^{\dagger}, \varphi_{2}^{\dagger}\right)$, and by comparison of the Majorana equations with the chiral representation we have the connections, $\phi_{ \pm}=\left(\phi_{1} \pm \phi_{2}\right) / 2$, and $\phi_{1,2}=\left(\phi_{+} \pm \phi_{-}\right)=(1 \pm S) \phi$. We insert these components in a four-component spinor that is again called $\psi^{\mathrm{C}}$. How does this state transform under the operation $\mathrm{C}=\gamma S$ ? We apply the charge-conjugation operation in its matrix form based on (7) on the above defined spinor, and by using $S^{2}=-1$ we obtain

$$
\mathrm{C} \psi^{\mathrm{c}}=\left(\begin{array}{cc}
0 & S \\
-S & 0
\end{array}\right) \cdot\left(\begin{array}{l}
(1+S) \phi \\
(1-S) \phi
\end{array}\right)=\psi^{\mathrm{c}}
$$

Again, the charge-conjugation operator leaves the eigen spinor $\psi^{\mathrm{C}}$ in the standard representation of the Dirac equation unchanged. Upon insertion of $\psi^{\mathrm{C}}$ in the standard Dirac equation, we obtain

$$
\begin{aligned}
i \frac{\partial}{\partial t}(\phi+S \phi)+i \sigma \cdot \frac{\partial}{\partial \boldsymbol{x}}(\phi-S \phi) & =m(\phi+S \phi) \\
\mathrm{i} \frac{\partial}{\partial t}(\phi-S \phi)+\mathrm{i} \boldsymbol{\sigma} \cdot \frac{\partial}{\partial \boldsymbol{x}}(\phi+S \phi) & =m(-\phi+S \phi)
\end{aligned}
$$

Thus it is readily verified that $\psi^{\mathrm{C}}$ solves (6), since $\phi$ and $S \phi$ by definition fulfill individually the respective Majorana Equation (13) and (14).

\section{Summary and Conclusions}

We reconsidered the two-component Majorana equation. By making use of the spin-flip operator, we derived this equation with a mass term in a novel and manifestly covariant way, which revealed its intimate relations to the Dirac equation in its chiral as well as standard form. The eigenfunctions of the two-component Majorana equation were also calculated.

The new mathematical approach employed here demonstrates for self-complex-conjugate states the complete equivalence of the complex Majorana equation with its two-component spinor $\phi$ and the Dirac equation, when being constrained to its four-component spinor solutions $\psi^{\mathrm{C}}$ which can be built up by the two Majorana spinors $\phi$ and $S \phi$. However, the complex Majorana equation does not guarantee, but in fact breaks one of the important basic field symmetries, namely charge exchange (represented here by the operator $S$ ), and thus for the CPT-invariant description of massive charged fermions the unconstrained complex Dirac equation is of course required.

The two-component Majorana equation, coming in two related forms (13) and (14) that are connected by a spin flip, represents the simplest possible covariant relativistic wave equation for a massive but uncharged fermion. These two forms represent the two irreducible representations of the Lorentz group in terms of Pauli spinors and matrices. Thus the Majorana equation can directly be derived by linearization of the quadratic relativistic energy-momentum relation, a procedure which 
leads to (11) without explicit recourse to the Dirac equation.

\section{References}

[1] E. Majorana, "Teoria Simmetrica Dell’ Elettrone E Del Positrone," Il Nuovo Cimento (1924-1942), Vol. 14, No. 4, 1937, pp. 171-184. doi:10.1007/BF02961314

[2] R. N. Mohapatra and P. B. Pal, "Massive Neutrinos in Physics and Astrophysics,” World Scientific, Singapore, 2004. doi:10.1142/9789812562203

[3] M. Fukugita and T. Yanagida, "Physics of Neutrinos and Applications to Astrophysics,” Springer, Berlin, 2003.

[4] M. Kaku, "Quantum Field Theory, A Modern Introduction,” Oxford University Press, New York, 1993.

[5] H. Weyl, "Elektron und Gravitation I," Zeitschrift für
Physik A Hadrons and Nuclei, Vol. 56, No. 5-6, 1929, pp. 330-352. doi:10.1007/BF01339504

[6] W. Pauli, "Zur Quantenmechanik des Magnetischen Elektrons," Zeitschrift für Physik A Hadrons and Nuclei, Vol. 43, No. 9-10, 1927, pp. 601-623. doi:10.1007/BF01397326

[7] P. B. Pal, "Dirac, Majorana and Weyl Fermions," arXiv:1006.1718v2 [hep-ph], 2010.

[8] P. M. A. Dirac, "The Quantum Theory of the Electron," Proceedings of the Royal Society of London. Series A, Containing Papers of a Mathematical and Physical Character, Vol. 117, No. 778, 1928, pp. 610-624.

[9] A. Das, "Lectures on Quantum Field Theory,” World Scientific, Singapore, 2008. doi:10.1142/9789812832870 